\title{
Development of QoS Evaluation Algorithm for MQTT Protocol with Reference to Threat Model
}

\author{
Shital Pawar, Suhas Patil
}

\begin{abstract}
MQTT protocol is publishing-subscribing model for IoT communication. In case of Quality of Services analysis, it is important to check the request and responses between publisher and subscriber. Any threat in communication channel is mostly leads to delay in operation. Hence, if we able to identify the delay parameter, we can suggest by means of QoS that there is a immediate need of security check for IoT system. As many IoT devices performed in unchecked, complicated, and often aggressive surroundings, safe-guarding IoT units present many different challenges. The key purpose for support quality degradation of IoT device interaction can be harmful attacks. Plenty of gadgets are often susceptible to port attacks/botnets hits, such as network attack events, which usually assessed by performing QoS Analysis. To start with factors affecting Quality of Services (QoS), in this paper we developed QoS evaluation algorithm "MQoS" for MQTT protocol and considered QoS-0 as an evaluation parameter. This paper refers the threat model which represents the flow of threats for proposed case study and can help to identify QoS by evaluating the possible communication threats. End-to-end device communication requests and responses are needed to be evaluated for large systems to get the actual QoS parameters for that system. For this reason the actual QoS tests will be conducted for third party applications.In this paper we presented results of MQTTV311 simulation for cooling sensor system.
\end{abstract}

Index Terms: IoT, QoS, Risk engineering, IoT device, MQTT

\section{INTRODUCTION}

Device monitoring programs frequently assist pressing out changes instantly to gadgets because well as controlling rollbacks if the upgrade procedure does not work out. They are capable to help to make sure that just authentic improvements are used by using digital putting your signature on.In huge level IoT systems, the intricacy of the system when it comes to the quantity of devices connected, and the range of products, applications, providers, and linking protocols involved, can make it difficult to identify when an event required place. Interesting in screening and ethical hacking to reveal vulnerabilities and applying protection cleverness and analytics to determine and inform

Revised Manuscript Received on October 30, 2019

* Correspondence Author

Shital Pawar*, Ph. D. Research Scholar, Bharati Vidyapeeth (Deemed to be University) College of Engineering, Pune,411043,India

Dr. Suhas Patil, Professor, Department of Computer Engineering, Bharati Vidyapeeth (Deemed to be University) College of Engineering, Pune,411043,India

(C) The Authors. Published by Blue Eyes Intelligence Engineering and Sciences Publication (BEIESP). This is an open access article under the CC BY-NC-ND license (http://creativecommons.org/licenses/by-nc-nd/4.0/) when occurrences happen.

Problems incorporate discovering which products had been contaminated, what information or services were used or destabilized and which end users had been affected, and after that selecting activities to cope with the situation. This is definitely required to assess QoS.

Gadget managers preserve a sign-up of gadget can be utilized to disable or isolate affected items until they can end up being patched. This feature can be especially essential for important devices this kind of as gateway devices in purchase to limit their potential to trigger damage or interruption, for example, by water damage the program with false data if they possess been sacrificed. Actions can be used undoubtedly utilizing a limitations electrical generator with recommendations centered on susceptibility control methods.

As proposed work considers IoT device request communication, to evaluate QoS it is necessary to identify the congestion period or packets loss etc. The IoT platform shows issues that incorporate detrimental individuality take advantage of the flow of data to and from network-connected things or modifying with gadgets requests themselves, which can provoke to the thievery of susceptible data and impairment of client security, collapse of internet operation through large-scale applied denial-of service attacks, and probable interruptions to significant facilities.

The Internet of Things (IoT) allows the integration of data from virtual and physical worlds. It entails smart items that can understand and respond to their environment in a range of commercial, industrial and home configurations. As the IoT expands the amount of linked devices, there can be the potential to enable cyber-attackers into the physical globe in which we live, as they seize on protection openings in these fresh systems. New security problems occur through the heterogeneity of IoT applications and products and their large-scale deployment $[1,2,3]$.

Content writer proposed the combined strategy to model a solitary optimization problem where the goal is definitely to jointly specify resources for request-level providers and for message agents, subject matter to common QoS limitations. In performing therefore, the joint strategy fits the system parts to assets obtaining into concern the models of interacting peers.

The IoT services need a number of Quality of (QoS) factors this kind of as group Services utilization, resource provisioning, work usage, program hold off, etc. The record technique for the offered model offers also been suggested and consequently the general overall performance evaluation of the technique can be confirmed when it comes to the QoS metrics [5] 
Various other studies reveal that, within the circumstances of IoT technologies, it is often the data that IoT gadgets generate that creates a comparable advantage. The author determined this as the "risk prize ratio", security and privacy risks increase as well [6].

Hence, in this analysis, by analyzing the attack graph (refer figure 1), a security analyst can assess the risks of potential intrusions and create effective defensive strategies.

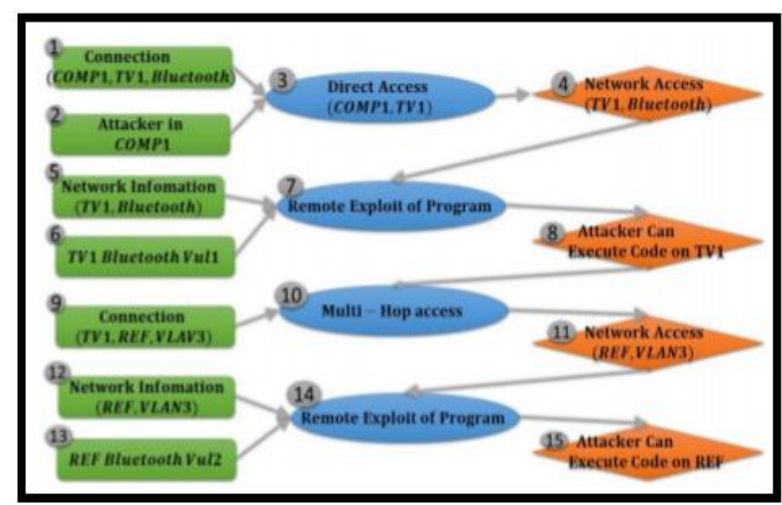

Figure 1- Illustration of Attack Graph [7]

For this, the arbitrary strolls and design theorem is definitely used. At first, the issue of physical assault recognition can be launched deployment technique, taking into consideration a affordable sensing model [11, 12]. The majority of these attacks are little alternatives of previously known cyber episodes. Nevertheless, the network utilized comprised merely of IoT units, many of which were the corresponding kind of device. The actual amount and circulation of the produced traffic make minimization extremely extravagant. Previous study strategy is definitely computationally effective, incurs much less connection over head and at the same period, provides a solid protection against numerous occasions such as, resource tiredness, Denial-of-Service, this increases the acknowledgement and the police arrest of this kind of episodes, restricting their harmful impact. The performance of the deep model is definitely completely in comparison with common machine learning technique and allocated assault recognition can be certainly evaluated against the central analysis technique. The primary issue is certainly to connect unfamiliar traffics to family members of known dangers. It can become a payload-based encryption plan that uses a basic four-way handshake system to confirm the identities of the taking part products [17].

Attack graphs are used to represent collections of complex multi-step strike scenarios seeing a business from an initial entry point to the most crucial resources. The aim of an author is to demonstrate the kinds of vulnerabilities that exist in home monitoring sensible cameras and to demonstrate their effects on users' security and privacy, by proposing a threat super model and a security and privacy analysis framework. Ranges of vulnerabilities are discovered with respect to the construction. Threat graphs have been used to estimate the protection risk score of organizational systems [16]. Topology robustness is certainly attained by degree difference and some functions. IoT gadgets introduce additional challenges to protection risk modeling through assault graphs, such as the diverse physical locations, a variety of short-range communication protocols, cyber-physical capabilities of the devices, mobility, etc. In all of this analysis, the structure of the regular IT network is normally analyzed, taking into account the vulnerabilities of workstations and servers. In this paper, the writer suggested a new security approach for the real-time IoT system. The results demonstrate (Refer number 1 above) (1) the contribution of the augmented strike graphs to quantifying the influence of IoT products used within the corporation on security, and (2) the effectiveness of the optimized IoT deployment [8].

Existing research scheme improved the performance of the topology of network without modifications in the structure of distributed nodes. Moreover, we observed that the GEA strategy is definitely capable to mis-classify all IoT malware samples as benign [9]. The attack chart analysis methodology includes three primary levels: (1) network and vulnerability scanning, (2) threat modeling. This study is definitely aimed at adopting a new strategy, deep learning, to cyber protection to allow the detection of events in the Internet of things. The outcome is discussed in detail and, demonstrating its efficiency [19]. Author carried a datasets testing for validation analyses. They can end up being implemented before the assault, during the strike (recognition), and after the attack [18]. The proposed technique is definitely evaluated using a real network with simulated deployment of IoT devices. The platform is definitely applied to give asset intelligent camcorders. The authors suggested a structure for IoT gadget protection modeling with the target of delivering all possible assault paths in the network, evaluating the security level, and assessing the efficiency of different defense strategies. It has also been shown that the deep model is definitely more effective in strike detection than its shallow countertop parts [14].

This recognition method can also reduce the detection range, increase recognition accuracy, and improve the robustness and scalability of the detection program [10]. Botnets such as Mirai have utilized insecure consumer IoT products to conduct distributed denial of services (DDoS) events on essential Internet infrastructure [9].

Furthermore, among the existing APT detection strategies, and almost all the features removed are local, which leads to the reality that the related methods possess poor scalability, hence reducing the precision. The author used attack graphs in association with IoT gadgets. This graph for the unidentified assault is definitely coordinated to a pre-known risk database; the GEA approach aims to preserve the efficiency and practicality of the produced adversarial sample through a careful embedding of a benign test to a destructive one. The purposeful of DDoS defenses is certainly to discover the assault as shortly as possible, and also to mitigate it.

In this work, Recently, DDoS events have been launched by zombie IoT devices in clever home networks. This motivates the development of brand-new techniques to find customer IoT strike traffic. And, at the same period, The IoT products are extremely susceptible to APT attacks. 
A light-weight recognition algorithm is developed for IoT products, which is normally structured on forecasting and turmoil theory to recognize flooding and DDoS events [15].

\section{PROPOSED METHODOLOGY}

As proposed research focusing on factors of QoS which gets affected by security issues of IoT system, the proposed methodology is used for threat mapping for QoS evaluation. Threat modeling can evaluate the QoS parameters like bit-rate, delay, packet dropping probability and jitter which intern supports proposed QoS model and can be used to enhance security. There are few QoS parameters that cannot become threat patterned and will be depend on how much it communicates, with the IoT system. As a future evaluation we will use QoS dataset for QoS model analysis. QoS of Threat modeling can end up being carried out at dataset communication stage of to ensure that the results can inform the style. Figure 2 shows block diagram of IoT control system flow as a representation of IoT system communication flow.

\section{A. Block Diagram}

Steps involved in QoS Threat Modeling are:

- Identification of QoS parameters: QoS parameters help you to focus the risk modeling activity.

- Decompose QoS Parameters: A detailed understanding of the QoS parameters should uncover more relevant threats and likely impact on QoS parameters.

- Identify risks: Make use of QoS information to identify threats relevant to your application scenario and context.

- Apply QoS: After evaluation of QoS parameters compare those with ideal system QoS parameters.

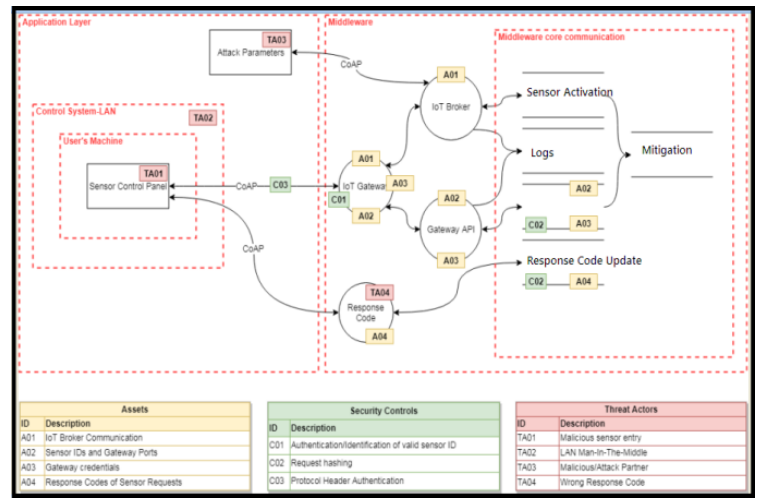

Figure 2 - Block diagram of IoT control system flow

To evaluate core QoS parameters for system (shown in figure 2), we represented attack tree in figure 4 below. This can help us to evaluate the parameters of each level of operation. Here is considered that there is a security threat in input request which may proceed through gateway port which can also be produce threat for malfunctioning of system sensors. To identify QoS parameters proposed algorithm MQOS (Mqtt QoS) is developed and simulated for MQTTv311. To generate fake attack we provided delay of $60 \mathrm{~ms}$. The assumed attack traverse through request port (1883) where there is a possibility to execute attack "Boat" of hacker. Following algorithm is developed for QoS-0 analysis.

\section{B. MQOS Algorithm}

To subscribe the incoming request:

1. Activate eclipse paho mqtt client

2. input: clientId, userdata, broker_url,

3. Mqttbroker_port=1883 // standard MQTT port

Retrieval Number F8161088619/2019@BEIESP

DOI: 10.35940/ijeat.F8161.088619

Journal Website: www.ijeat.org

\section{String protocol = "MQTTv311"}

5. String transport = "tcp" // Type of MQTT protocol

6. set flag $=1 / /$ if incoming request then flag will be ' 1 ',

7. array topic [] // this is sensor event information

8. array msg_count=[] // all incoming messages will be stored

9. array qos [] // stores QoS code i.e. QoS-0 or QoS-1

10. clientId con $=$ connection() $/ /$ call for client connection

11. if qos $<0 \|$ qos $>2$

12. show ValueError

13. if msg_count $<1 / /$ checking message validity

14. callback to pub module

To publish the processed response

15. getHost () // connect to host

16. getMqttbroker_port() //open MQTT broker port

17. getClientId()

18. setSessionAlive $=60 / /$ setting of delay to show security flaw

19. redirectProtocol (paho.Mqttv311) //Eclipse paho request

20. if array topic $!=0 \& \&$ array msg_count $!=0$

21. setUserdata () \&\& setQoS () // identification of QoS parameter

22. else

23. session ("expired")

\section{Flowchart}

The QoS-0 flow is depicted for MQTT protocol in figure 3 below. This shows the publisher request flow to broker where broker publishes the message to subscriber. In case of QoS-0, there is no acknowledgement is provided. Publisher will successfully publish message if there is no threat or in case of any threat, publisher will lead to session expiry call and deletes the message.

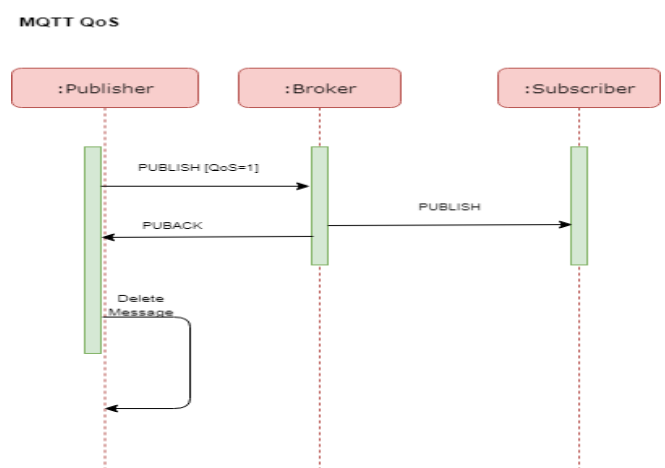

Figure 3 - Proposed QoS-0 Analysis request flow for MQTT

Sensing quality monitoring relates to the overall functionality of QoS subscription monitoring and management and will be based on the IoT protocol communication. Data received from sensors which have been published during a predefined time window is needed to be analyzed for QoS analysis. Refer figure 3 and figure 4 for Proposed QoS Analysis for MQTT request flow. 


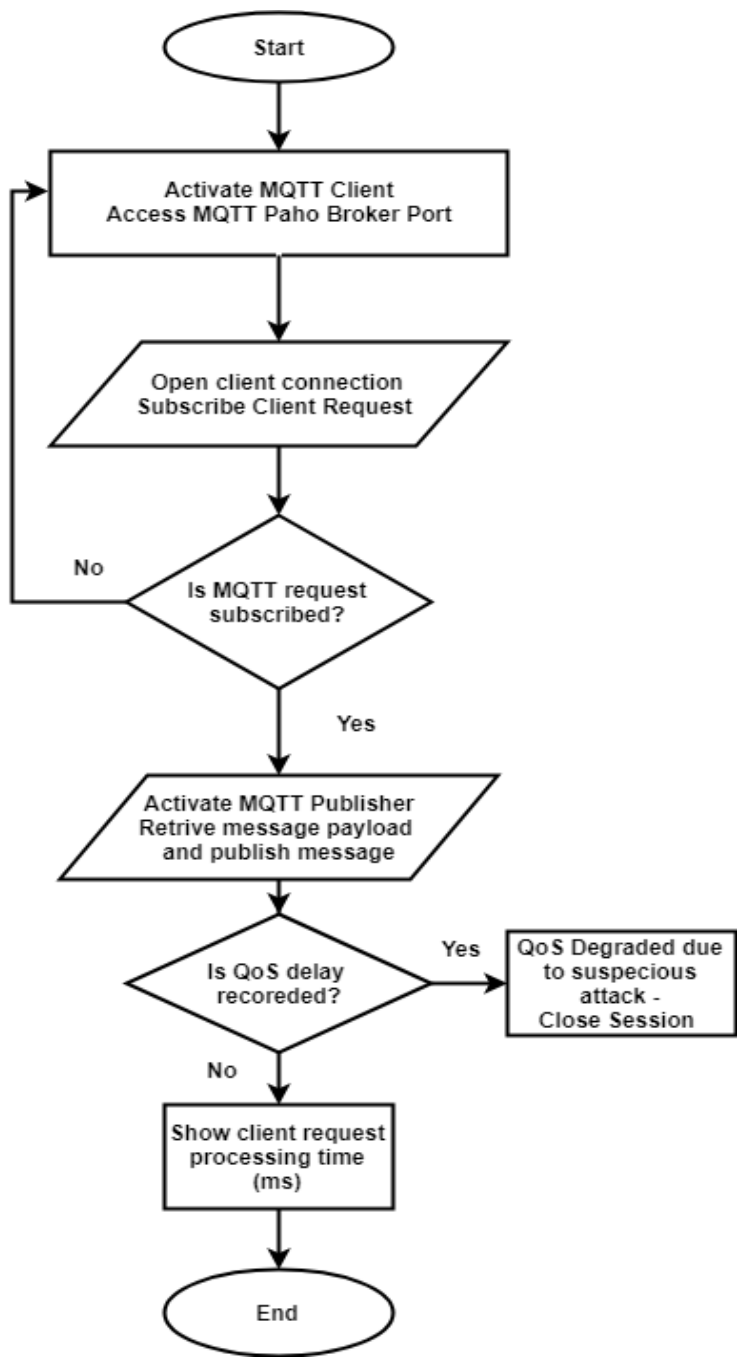

Figure 4 -Flowchart for QoS proposed MQoS Algorithm

\section{RESULT ANALYSIS}

Thus, IoT system performance may become unstable which leads to request/response failure in streamlined protocol communication. Hence, QoS model can identify the performance degradation by means of differences in QoS parameter values. Further, in section III-A of this paper we presented a case study for temperature sensor to depict the malfunctioning of sensors.

In proposed case of Message Queue Telemetry Transport (MQTT) protocol is considered. To analyze the MQTT QoS-0, the attack model is depicted here in figure 5. This attack model is developed with assumption that IoT system receiving incoming attack request which causes delay in actual request processing.

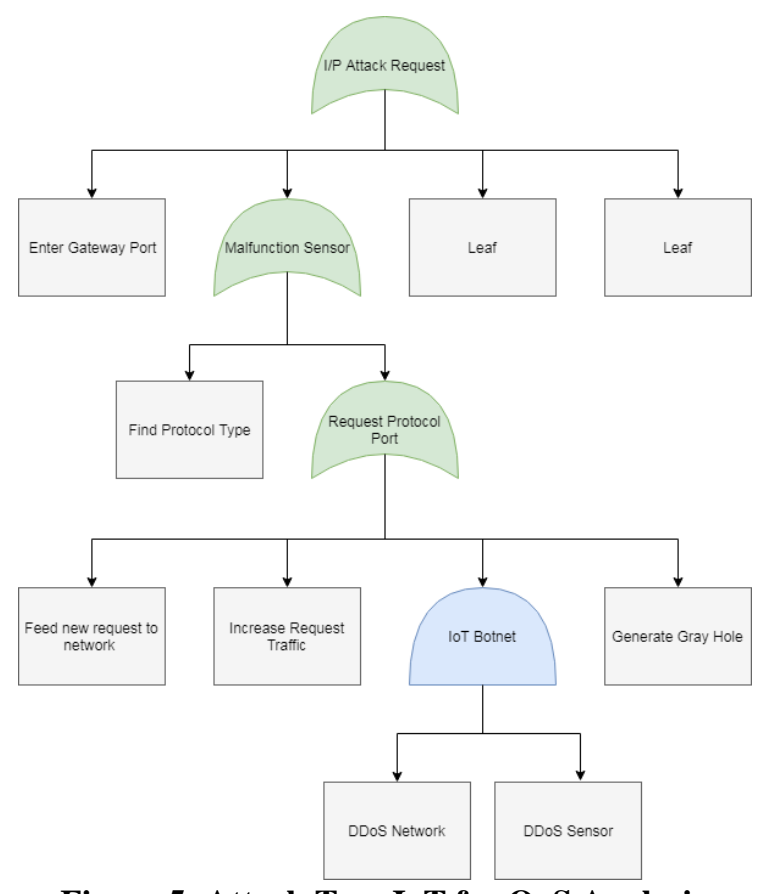

Figure 5- Attack Tree IoT for QoS Analysis

\section{A. Case Discussion}

The following safe operation logic could be applied in order to demonstrate proof of the concept. Each condition generates an error message: -If cooler thermal importance $<15$ -If cooler thermal importance $>20$ -If thermostat switching routine $>30$ minutes -If cooler thermal importance "Tc"( in thermostat switching cycle ) >30 -If cooler thermal worth $>20 \mathrm{C}$ initially Therefore, Different types of threats for an IoT chilling system generally consist of:

- Blocking of data sent between the remote control panel and the IoT air conditioning program. This may be used to disturb the procedures of the IoT cooling system. - Manipulation of data transmitted between the IoT remote control panel and the IoT chilling program. This could be utilized to maliciously change the operation of the IoT air conditioning system. The unauthorized use of IoT cooling programs could involve harmful procedures of the IoT chilling

system.

- Unauthorized adjustment of stuck software program in the IoT air conditioning program. The assault shrub protection levels in the multi-tiered security strategy defined would reverse the various types of potential protection breaches: - the utilization of encryption to reduce the possibility of interception or manipulation of data sent. The usage of individual conversation stations for data transmitting from the remote panel to the IoT cooling system and from the IoT chilling program to the consumer. This could decrease the likelihood of obstructing or interruption. The QoS-0 is represented in figure 3 above with respect to case discussed in this paper. 


\section{B. DDoS Attack}

This attack occurs when multiple systems deluge the bandwidth or resources of a targeted system, generally one or even more internet servers. Such an assault can be frequently the result of multiple compromised systems (for example, a botnet [13] where QoS gets degraded due to flooding the targeted program with visitors. Due to absence of fundamental protection controls, IoT devices are soft targets for cyber criminals and other aggressors. This implies that they can be very easily hacked and added to botnets, which are used to launch DDoS.

This can be evaluated by analyzing the throughput parameter of QoS [21]. In an extremely simplified description, CoAP can be extremely identical to HTTP, it functions on best of UDP, Simply like HTTP is used to carry data and instructions between a customer and a machine, but without requiring the same quantity of assets, which makes it ideal for today's increasing influx of IoT devices. In the world of DDoS [20] attacks, this can range from 10 to 50 , Internet bots, once contaminated, botnets have been utilized period and once again in DDoS events around the globe and their figures are just raising (Refer figure 6).

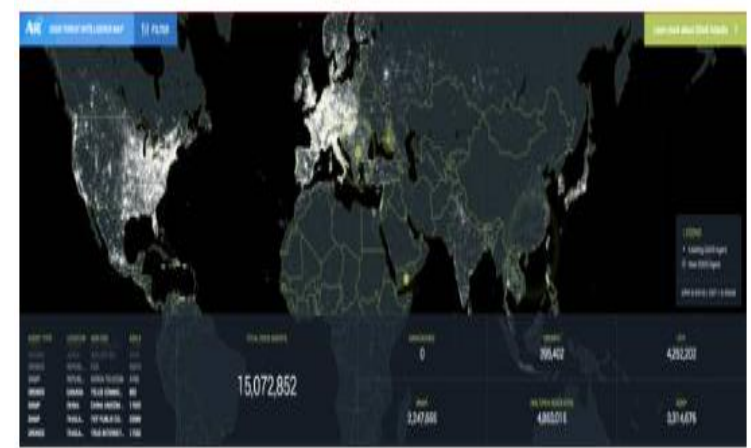

Figure 6 - A10 DDoS Threat Intelligence Map (Source: A10 Networks, Inc)

Hence, evaluation and processed information about potential or current events threatens a system. This intelligence can be utilized to identify vulnerable spots and proactively evaluate defence without losing precious periods and assets.

QoS-0 implies the publisher request traverse to broker and broker forwards request to subscriber to publish intended request. This is one way MQTT protocol journey with zero acknowledgement response. The end-to-end delay is determined in terms of 'ms'. Hence, the QoS-0 is evaluated for MQTT IoT protocols.

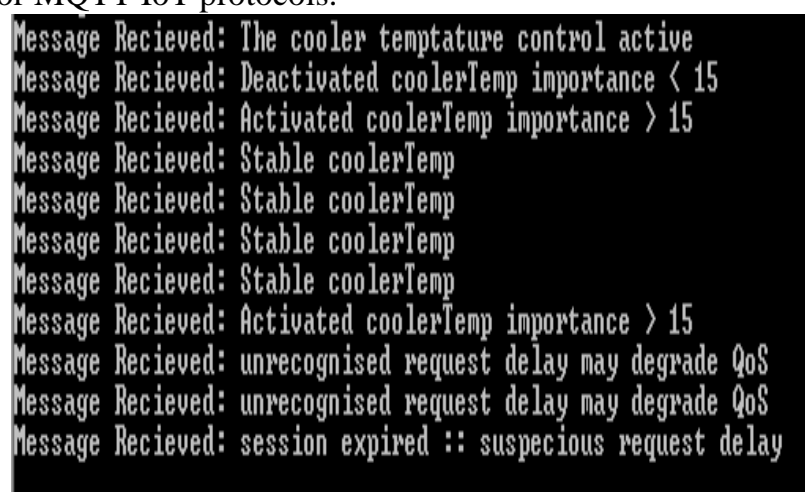

Figure 7 - MQOS: Message Subscription requests

Retrieval Number F8161088619/2019@BEIESP

DOI: 10.35940/ijeat.F8161.088619

Journal Website: www.ijeat.org
The MQOS algorithm with reference to case discussed is discussed in previous section-II of this paper to understand the system operations. The proposed algorithm works in pub-sub sequence as MQTT protocol works for subscribing the published requests. Hence, we provided the simulation for request generation. The QoS-0 is a one way request processing without acknowledgment. So, the end-to-end delay is calculated with explicitly generated delay and without explicit delay. Figure-7 above shows the result of pub-sub request flow for temperature control of cooler.

Table 1- MQTT request results

\begin{tabular}{|l|l|l|}
\hline $\begin{array}{l}\text { Cooler } \\
\text { Temperature } \\
\text { (Ct) }\end{array}$ & $\begin{array}{l}\text { Delay } \\
\text { Recorded? } \\
\text { (Yes/No) }\end{array}$ & Time (ms) \\
\hline $\mathrm{Ct}<15$ & No & 21 \\
\hline $\mathrm{Ct}>15$ & No & 23 \\
\hline $\mathrm{Ct}<15$ & Yes & 81 \\
\hline $\mathrm{Ct}>15$ & Yes & 83 \\
\hline
\end{tabular}

As per execution, results shown in table-1 above. The normal operation recorded for acceptable temperature control for importance 15 with 21 and 23 ms respectively. Whereas delay in request processing is recorded with $60 \mathrm{~ms}$ which means the QoS-0 is degraded due to suspicious attack and need to be evaluated by system engineers.

\section{CONCLUSION}

QoS parametric evaluation is important for safely handling IoT applications and solutions. Authentication security by default are available for protection which allows to keep data privacy and integrity, while producing highly obtainable IoT data, apps, and providers. But, in case of any attack, it is necessary to identify the parameter which degrades due to attack. DDoS attacks are represented as a reference IoT attacks for understanding worldwide attacks incidences. This paper evaluated QoS-0 for cooling system with simulation of MQTTv311 protocol and threat is generated with delay provision during publishing broker message. The MQTT broker request pub-sub model is demonstrated. The algorithm and flowchart represents the flow for the MQTT QoS-0 identification. Further, as a future research, QoS-1 modeling is necessary to evaluate such attacks and to provide key reason for performance degradation.

\section{REFERENCES}

1. Radanliev, Petar, et al. "Definition of Internet of Things (IoT) Cyber Risk Discussion on a Transformation Roadmap for Standardisation of Regulations Risk Maturity Strategy Design and Impact Assessment." arXiv preprint arXiv:1903.12084(2019).

2. Radanliev, Petar, et al. "Standardisation of cyber risk impact assessment for the Internet of Things (IoT)." arXiv preprint arXiv:1903.04428 (2019).

3. Peros, Stefanos, et al. "Dynamic QoS support for IoT backhaul networks through SDN." 2018 Third International Conference on Fog and Mobile Edge Computing (FMEC). IEEE, 2018.

4. Costa, Fábio M., et al. "Cross-layer QoS-Aware Resource Allocation for IoT-Enabled Service Choreographies." Proceedings of the 5th Workshop on Middleware and Applications for the Internet of Things. ACM, 2018. 
5. Maiti, Prasenjit, et al. "Mathematical Modeling of QoS-Aware Fog Computing Architecture for IoT Services." Emerging Technologies in Data Mining and Information Security. Springer, Singapore, 2019. 13-21.

6. Jalali, Mohammad S., et al. "The Internet of Things Promises New Benefits and Risks: A Systematic Analysis of Adoption Dynamics of IoT Products." IEEE Security \& Privacy 17.2 (2019): 39-48.

7. Agmon, Noga, Asaf Shabtai, and Rami Puzis. "Deployment Optimization of IoT Devices through Attack Graph Analysis." arXiv preprint arXiv:1904.05853 (2019).

8. Nia, Mehran Alidoost, et al. "Detecting new generations of threats using attribute-based attack graphs." IET Information Security (2019).

9. Abusnaina, Ahmed, et al. "Examining Adversarial Learning against Graph-based IoT Malware Detection Systems." arXiv preprint arXiv:1902.04416 (2019).

10. Ma, Zhen, Qiang Li, and Xiangyu Meng. "Discovering Suspicious APT Families Through a Large-Scale Domain Graph in Information-Centric IoT." IEEE Access 7 (2019): 13917-13926.

11. Qureshi, Talha Naeem, et al. "Enhanced Robustness Strategy for IoT in Smart Cities Based on Data Driven Approach." Workshops of the International Conference on Advanced Information Networking and Applications. Springer, Cham, 2019.

12. Jan, Mian Ahmad, et al. "A payload-based mutual authentication scheme for Internet of Things." Future Generation Computer Systems 92 (2019): 1028-1039.

13. Meidan, Yair, et al. "N-BaIoT—Network-Based Detection of IoT Botnet Attacks Using Deep Autoencoders." IEEE Pervasive Computing 17.3 (2018): 12-22.

14. Diro, Abebe Abeshu, and Naveen Chilamkurti. "Distributed attack detection scheme using deep learning approach for Internet of Things." Future Generation Computer Systems 82 (2018): 761-768.

15. Halder, Subir, Amrita Ghosal, and Mauro Conti. "Efficient Physical Intrusion Detection in Internet of Things: A Node Deployment Approach." Computer Networks (2019).

16. Alharbi, Rana, and David Aspinall. "An IoT analysis framework: An investigation of IoT smart cameras' vulnerabilities." (2018): 47-10.

17. Kuzminykh, Ievgeniia, and Anders Carlsson. "Analysis of Assets for Threat Risk Model in Avatar-Oriented IoT Architecture." Internet of Things, Smart Spaces, and Next Generation Networks and Systems. Springer, Cham, 2018. 52-63.

18. Bhardwaj, Ketan, Joaquin Chung Miranda, and Ada Gavrilovska. "Towards iot-ddos prevention using edge computing." \{USENIX\} Workshop on Hot Topics in Edge Computing (HotEdge 18). 2018.

19. Procopiou, Andria, Nikos Komninos, and Christos Douligeris. "ForChaos: Real Time Application DDoS Detection Using Forecasting and Chaos Theory in Smart Home IoT Network." Wireless Communications and Mobile Computing 2019 (2019).

20. Doshi, Rohan, Noah Apthorpe, and Nick Feamster. "Machine learning ddos detection for consumer internet of things devices." 2018 IEEE Security and Privacy Workshops (SPW). IEEE, 2018.

21. Shital Pawar, Suhas Patil "Current Security Challenges in Internet Things" International Journal of Electronics and Computer Engineering, vol 7, issue 2, (2019): 2399-2401.

\section{AUTHORS PROFILE}

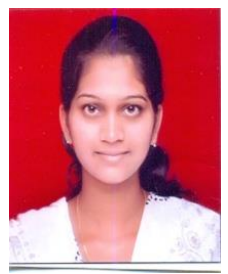

Shital Pawar received the B.E. degree and M. Tech degree in Computer Engineering from Bharati Vidyapeeth Deemed University, Pune and pursuing $\mathrm{Ph}$. D. degree from Bharati Vidyapeeth (Deemed to be University) College of Engineering, Pune. She is currently working as an Assistant Professor in Computer Engineering department of Bharati Vidyapeeth's College of Engineering for Women, Pune. Her research interests include Internet of

Things.

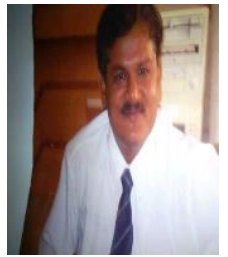

Suhas Patil received the B.E. degree in Computer Engineering from Shivaji University, W.I.T Solapu in 1989, the M. E. degree from Govt. College of Engg, University of Pune in 1992 and the Ph. D degree from Bharati Vidyapeeth Deemed University, Pune in 2009. He is currently working as the Professor in Computer Engineering Department of Bharati Vidyapeeth (Deemed to be University) College of Engineering, Pune. His research interests include Operating System and Distributed System. He has published more than 268 research papers in reputed peer reviewed National and International Journals and Conferences. His biography published in Marquis who's who 2011 Edition.
Published By:
Blue Eyes Intelligence Engineering \& Sciences Publication 